\title{
Lendatus, a new genus of Xanthopygina (Coleoptera: Staphylinidae: Staphylininae) with description of three new species
}

\author{
Stylianos Chatzimanolis ${ }^{\text {Corresp. } 1}$ \\ ${ }^{1}$ Department of Biology, Geology and Environmental Science, University of Tennessee at Chattanooga, Chattanooga, Tennessee, United States \\ Corresponding Author: Stylianos Chatzimanolis \\ Email address: stylianos-chatzimanolis@utc.edu
}

A new genus of Xanthopygina rove beetles is described here as Lendatus gen. nov. The new genus includes three new species: L. bolivianus sp. nov., described from Bolivia, $L$. philothalpiformis sp. nov. described from Costa Rica and Panama, and L. platys sp. nov. described from Bolivia, Colombia, Ecuador and Peru. Lendatus belongs to the Isanopus group of genera of Xanthopygina and can distinguished from all the genera based on the unique punctation on the pronotum and the long apical setae of the paramere. A key to the three species of Lendatus along with photographs and illustrations is provided for the identification of species. 
1 Lendatus, a new genus of Xanthopygina (Coleoptera: Staphylinidae:

2 Staphylininae) with description of three new species.

3

4

5 Stylianos Chatzimanolis

6

7 Department of Biology, Geology and Environmental Science, University of Tennessee at Chattanooga,

8615 McCallie Ave., Dept. 2653 Chattanooga, TN, USA

9

10 Corresponding Author:

11 Stylianos Chatzimanolis

12 Email address: stylianos-chatzimanolis@utc.edu

13

14

15

16

17

18

19

20

21

22

23

24

25

26

27

28

29

30

31

32

33

34

35

36

37

38

39

40

41 
42

43

44

45

46

47

48

49

50

51

52

53

54

55

56

57

58

59

60

61

62

63

64

65

66

67

68

69

70

71

72

73

74

75

76

77

78

79

80

81

82

83

84

85

86

\section{Abstract}

A new genus of Xanthopygina rove beetles is described here as Lendatus gen. nov. The new genus includes three new species: L. bolivianus sp. nov., described from Bolivia, $L$. philothalpiformis sp. nov. described from Costa Rica and Panama, and L. platys sp. nov. described from Bolivia, Ecuador and Peru. Lendatus belongs to the Isanopus group of genera of Xanthopygina and can distinguished from all the genera based on the unique punctation on the pronotum and the long apical setae of the paramere. A key to the three species of Lendatus along with photographs and illustrations is provided for the identification of species.

\section{Introduction}

Xanthopygina is a diverse group of mostly neotropical rove beetles that includes (before the publication of this paper) 29 genera. In the latest phylogenetic analyses of the subtribe, Chatzimanolis \& Brunke (2019) were able to examine all genera of Xanthopygina and identified the major lineages of the subtribe, One of them was the Isanopus group of genera, which included four genera: Zackfalinus Chatzimanolis (Chatzimanolis 2012) as the sister group of Peripus Chatzimanolis \& Hightower (Chatzimanolis \& Hightower 2019; identified in the phylogeny paper as genus 5), and Isanopus Sharp (Chatzimanolis 2008) as the sister group of genus 2. That genus 2 is described in this paper as the new genus Lendatus Chatzimanolis and includes three new species.

The sister group relationship between Isanopus and Lendatus was first identified by Chatzimanolis (2014) in the first molecular phylogeny of the subtribe, where Lendatus was presented in that phylogeny as 'undescribed genus'. Delimiting new taxa, especially above the species level is not straightforward and ideally one should have multiple lines of evidence before proposing formal taxonomic names. While I had strong molecular evidence that Lendatus is indeed a new genus for quite some time, I did not feel comfortable describing Lendatus as new taxon until the completion of the morphological analysis of the subtribe that included all described genera and a number of undescribed ones.

\section{Materials \& Methods}

Specimen preparation, study and photography followed other recently published papers on Xanthopygina (e.g., Chatzimanolis \& Hightower 2019). Dissected aedeagi were placed in small glass vials filled with glycerin and pinned underneath the specimen. I took the following measurements: HL: head length, at middle, from the anterior margin of frons to the nuchal ridge; HW: Head width, the greatest width, including the eyes; PL: pronotum length, at middle; PW: pronotum width, greatest width; EL: elytra length, measured in lateral view from the anterolateral angle of the elytra to the apex of the elytra; however, I used these measurements only proportionally (e.g., PW/PL). As a surrogate of total body length, I used forebody length (FL), measured by adding HL+PL+EL. I examined specimens using an Olympus ZX10 stereomicroscope and I took photographs using a Canon 40D camera equipped with a MP-E 65

Peer] reviewing PDF | (2019:07:39356:1:1:NEW 16 Sep 2019) 
$87 \mathrm{~mm}$ macro lens on a Cognisys StackShot $3 \mathrm{X}$ macro rail and controller (https://www.cognisys-

$88 \mathrm{inc.com} /$ products/stackshot/stackshot.php). I automontaged images using Helicon Focus Pro

89 6.7.1 (http://www.heliconsoft.com/heliconsoft-products/helicon-focus/). I removed the

90 background of photographs using Fluid Mask 3 (https://www.vertustech.com). Type labels are

91 separated by a slash ' $\%$. Text within brackets [ ] is explanatory and was not included in the

92 original label. Generic description was extracted from the matrix in Chatzimanolis \& Brunke

93 (2019) with addition of a few other characters. I produced maps using the online program

94 SimpleMappr (Shorthouse 2010). In this paper, I used the phylogenetic species concept of

95 Wheeler \& Platnick (2000) to delimit different species. Datasets for each species in DarwinCore

96 format are available online at https://figshare.com/authors/Stylianos_Chatzimanolis/384794.

97

98

99

100

101

102

103

104

105

106

107

108

109

110

111

112

113

114

115

116

117

118

119

120

121

122

123

124

125

126

127

128

129

130

131

I examined specimens from the following institutions:

BMNH The Natural History Museum, London, UK (M. Barclay).

CMNC Canadian Museum of Nature, Ottawa, ON, Canada (R. Anderson).

CMNM Carnegie Museum of Natural History, Pittsburgh, PA, USA (R. Davidson).

CNC Canadian National Collection, Ottawa, ON, Canada (A. Brunke).

CRO G. de Rougemont collection, Oxford, UK (G. de Rougemont).

DEBU University of Guelph Insect Collection, Guelph, ON, Canada (S. Marshall).

FMNH Field Museum of Natural History, Chicago, IL, USA. (C. Maier).

MNCR-A National Museum of Costa Rica, San José, Costa Rica (A. Ruiz).

MUSM Universidad Nacional Mayor de San Marcos, Museo de Historia Natural,

Lima, Peru (D. Silva).

NHMD Natural History Museum of Denmark, University of Copenhagen,

Copenhagen, Denmark (A. Solodovnikov).

SEMC Snow Entomological Collection, Biodiversity Institute, University of

Kansas, Lawrence, KS, USA (Z. Falin).

UNSM University of Nebraska State Museum, Lincoln, NE, USA (B. Ratcliffe).

UTCI The University of Tennessee at Chattanooga, Chattanooga, TN, USA

(S. Chatzimanolis).

Please note that several of the specimens currently deposited in SEMC will be transferred to MUSM per previous institutional/collecting agreements.

The electronic version of this article in Portable Document Format (PDF) will represent a published work according to the International Commission on Zoological Nomenclature (ICZN), and hence the new names contained in the electronic version are effectively published under that Code from the electronic edition alone. This published work and the nomenclatural acts it contains have been registered in ZooBank, the online registration system for the ICZN. The ZooBank LSIDs (Life Science Identifiers) can be resolved and the associated information viewed through any standard web browser by appending the LSID to the prefix http://zoobank.org/. The LSID for this publication is urn:lsid:zoobank.org:pub:0612FF19-38E8-4072-AF740EB16165841. The online version of this work is archived and available from the following digital repositories: PeerJ, PubMed Central and CLOCKSS.

132 
133

134

135

136

137

138

139

140

141

142

143

144

145

146

147

148

149

150

151

152

153

154

155

156

157

158

159

160

161

162

163

164

165

166

167

168

169

170

171

172

173

174

175

176

177

178

\section{Results}

Taxonomy

Lendatus Chatzimanolis, new genus

(Figs. 1, 2, 3, 4, 5, 6, 7, 8, 9)

urn:Isid:zoobank.org:act:73EEC4F3-E35B-4E67-9FAA-5C8C14222ABB

Type Species. Lendatus platys, new species, here designated.

Diagnosis. Lendatus belongs to the Isanopus group of genera (see Chatzimanolis and Brunke 2019 for characters differentiating all genera in the Isanopus group) based on the following morphological characteristics: basal transverse carina on sternum 3 acutely pointed medially; lack of dense meshed microsculpture on sterna 5-7 (Fig. 4C); antennomeres 8-10 quadrate or elongate (Fig. 3E); and mesocoxae moderately to strongly separated (Fig. 4B). Lendatus was recovered as the sister group to Isanopus (Chatzimanolis 2014; Chatzimanolis and Brunke 2019) and the sister group relationship is supported by the following morphological characteristics (besides the molecular data supporting that relationship): coarse punctures impressed in flange at posterior angle of pronotum (Fig. 2); and lateral area of basal transverse carina on sternum 3 sinuate. Synapomorphies for Lendatus include: apical setae on paramere long, produced over the median lobe (Figs. 5, 6, 7), longer than any other Xanthopygina genus; and distribution of punctures on disc of pronotum split into anterior and posterior parts by diagonal longitudinal line, a unique character state in Xanthopygina. Additional characteristics that can distinguish Lendatus from Isanopus include: paramere not extremely reduced (as in Isanopus) and tarsomeres of middle and hind legs not enlarged and lobed (as in Isanopus).

Some species of Oligotergus Bierig may look superficially similar to Lendatus, but species in that genus typically lack the characteristics of the Isanopus group. Additionally, $L$. philothalpiformis has the same color pattern with some Philothalpus Kraatz species but Philothalpus can be easily distinguished by the presence of a pair of accessory ridges on the anterior basal transverse carina of tergum 3 (see Chani-Pose et al. 2018).

Description. Habitus as in Fig. 1. Body medium-sized, forebody 4.6-5.8 mm long; without long bristle-like setae. Coloration of head and pronotum dark brown to black with metallic overtones or bright reddish-orange; elytra dark metallic green, blue or purple; abdomen dark brown or reddish brown to dark brown.

Head (Fig. 2) shape rectangular; head length in comparison to pronotum shorter to subequal. Eye size relative to length of head large, more than 3/4 length of head. Postclypeus in comparison to frons not deflexed, anterior margin more or less straight. Middle of epicranium impunctate but with microsculpture. Postmandibular ridge laterally; with deep punctures demarcating raised postmandibular ridge dorsolaterally present. Gular sutures not joined before neck extended close to each other at base of head capsule. Nuchal ridge present. Neck disc punctures sparse.

Antennae (Fig. 3E), antennomere 1 same width or slightly wider than 2 . Antennomere 3 elongate, three times as long as wide; antennomere 4 with tomentose pubescence; antennomere 6 with curved, distinctly longer and thicker subapical setae than other macrosetae, forming circlet;

PeerJ reviewing PDF | (2019:07:39356:1:1:NEW 16 Sep 2019) 
179

180

181

182

183

184

185

186

187

188

189

190

191

192

193

194

195

196

197

198

199

200

201

202

203

204

205

206

207

208

209

210

211

212

213

214

215

216

217

218

219

220

221

222

antennomeres 1-11, cylindrical, longer than wide; antennomeres 8-10 symmetrical; antennomeres 5-10 without club; antennomere 11 in males subequal to 10 .

Mouthparts with labrum having broad U-shaped emargination, lobes strongly separated. Mandibles (Figs. 3A-B) relative length typical (i.e. closed mandible not extending beyond margin lateral margin of head); without asymmetrical torsion. Mandibles in dorsal view curved from apical half; in lateral view straight; left and right mandibles each with one tooth. Maxilla (Fig. 3D) with galea much shorter than palpus; maxillary palpus with $\mathrm{P}_{3}$ distinctly shorter than $\mathrm{P}_{2}$; $\mathrm{P}_{4}$ distinctly longer than $\mathrm{P}_{3} ; \mathrm{P}_{4}$ not dilated. Hypopharynx and labial palpi as in Fig. 3C; labial palpus $\mathrm{P}_{3}$ widest before apex, without long dense setae on entire lateral sides. Ligula small, entire. Mentum with alpha setae present; hypostomal cavity present, moderately delimited.

Pronotum (Fig. 2) shape of lateral margins in dorsal view posteriad of midpoint straight to sinuate (except L. platys convex); anterior angles in dorsal view not strongly acuminate and produced laterad. Pronotum near anterolateral angles without raised impunctate spots; anterolateral corners with punctation; disc of pronotum with punctation split into anterior and posterior parts by diagonal longitudinal line of punctures; with coarse punctures impressed in flange at posterior angle of pronotum; with microsculpture. Pronotum subquadrate; narrower than head at widest points. Hypomeron (Fig. 4A) with superior marginal line continuous to anterior margin; superior marginal line without deflection under anterior angles in ventral view; inferior marginal line continued as a separate entity beyond anterior pronotal angles and curving around them. Postcoxal process absent. Basisternum slightly longer than furcasternum; basisternum with pair of macrosetae situated far from anterior margin of prosternum.

Elytra without contiguous polygon-shaped meshed microsculpture or patches of white setae. Elytral setae not reduced, easily seen at low magnification (e.g., 40x). Mesoventrite (Fig. 4B) with anterior margin forming "lip"; without median carina; mesoventral process triangular; process extended distally to distance about $2 / 5$ between mesocoxae. Metaventrite (Fig. 4B) with large punctures; metaventral processes, small, rounded, triangular, extended to beginning of metacoxae.

Legs with tarsal segmentation 5-5-5; prefemora without lateroventral apical spines; protarsi with modified pale (adhesive) setae ventrally; tarsomeres 1-4 of protarsi dorsoventrally flattened. Mesocoxae (Fig. 4B) moderately separated; intercoxal area distinctly recessed compared to mesoventrital process. Metacoxae without coxal shield; metatibia without thick and long apical spurs but with smaller spurs and spines. Meso/metatarsi without asymmetrically lobed tarsomeres 1-4; tarsomeres 3-5 of metatarsi with chaetotaxy developed only at margins of dorsal surface, dorsal surface of tarsomeres glabrous along midline. Pretarsal claws with empodial setae.

Abdomen (Figs. 4C-D) with protergal glands having well-developed acetabula. Anterior basal transverse carina on terga 3-5 without pair of accessory ridges; tergum 3 without posterior basal transverse carina and without curved carina (arch-like) on disc; center of tergum 5 with punctation; posterior half of tergum 5 in lateral view not appearing bulging. Sternum 3 with acutely pointed basal transverse carina medially; laterally basal transverse carina sinuate; basal transverse carina absent on sternum 4; sternum 5 without dense, meshed microsculpture anterolaterally; sternum 7 with sparse punctation laterally. Males with secondary sexual structures (emargination medially on sterna 7 and 8); without porose structure. Females without obvious secondary sexual structures.

Peer] reviewing PDF | (2019:07:39356:1:1:NEW 16 Sep 2019) 
225

226

227

228

229

230

231

232

233

234

235

236

237

238

239

240

241

242

243

244

245

246

247

248

249

250

251

252

253

254

255

256

257

258

259

260

261

262

263

264

265

266

267

268

269

Aedeagus as in Figs. 5-7; with long median lobe and single paramere; paramere with sensory peg setae and long apical setae; median lobe with single subapical tooth; median lobe without apical tooth, carina or paired apex. Spermatheca not sclerotized.

Etymology. The name is in honor of my dear friends Dr. Ntina Karametsi, Dr. Lia Koutelou, Mr. Dimitris Kotsis, Dr. Tania Patsialou and Dr. Eleni Zika. The name is made up from a combination of letters from the first names. The name is masculine.

Habitat. Collected in lowland tropical rainforests and mid-elevation cloud forests using a variety of trapping techniques and by shifting leaf litter. The genus most likely inhabits the leaf litter.

\section{Lendatus bolivianus Chatzimanolis, new species \\ (Figs. 1A, 2A, 5, 8) \\ urn:Isid:zoobank.org:act:14E6C64D-E882-41D3-85DA-75930F62DCF1}

Type material. Holotype, here designated, male, "Bolivia: La Paz, $9.4 \mathrm{~km}$ E. Chulumani, ApaApa, $2400 \mathrm{~m}, 16^{\circ} 20.99 \mathrm{~S} 67^{\circ} 30.30 \mathrm{~W}$ [-16.349833, -067.505], 17.i.2001, R. Anderson, upper yungas litter, BOLA01-002" / "SM0459200 [barcode label]" / "HOLOTYPE Lendatus bolivianus Chatzimanolis, des. Chatzimanolis 2019". In the collection of SEMC.

Paratypes. Six; one with same locality label as holotype and barcode label SM0459190 (1 우 SEMC); "Bolivia: La Paz Prov. Chulumani, $9.2 \mathrm{~km}$ E of, $2300 \mathrm{~m}, 16^{\circ} 20.59 \mathrm{~S} 67^{\circ} 30.18 \mathrm{~W}$ [16.3431667, -67.503], 19-21 Jan[nuary] 2001, J. S. Ashe, R. S. Hanley, BOL1AH01 039 ex:

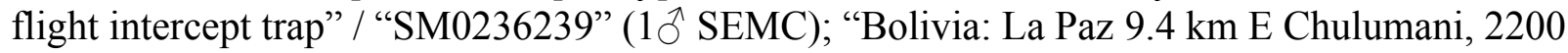
$\mathrm{m}, 16^{\circ} 20.99 \mathrm{~S} 67^{\circ} 30.30 \mathrm{~W}$ [-16.349833, -067.505], 19-21.i.2001, J. S. Ashe, R. S. Hanley, BOL1AH01 038 ex: flight intercept trap" / SM0574084, SM236231 (1우 SEMC; 1 ㅇ UTCI ); "Bolivia: Chulumani, Apa-Apa forest, 16²1'S, 67³0'W [-16.35, -67.5], 12-14.xi.2007, 2000 $\mathrm{m}$, shifting forest litter, V. Grebennikov leg." (1ㅇ, $\left.10^{\uparrow} \mathrm{NHMD}\right)$. All paratypes with label "PARATYPE Lendatus bolivianus Chatzimanolis, des. Chatzimanolis 2019".

Diagnosis. Lendatus bolivianus and L. platys can be distinguished from L. philothalpiformis by the coloration of head and pronotum (dark brown to black in L. bolivianus and L. platys; bright reddish-orange in L. philothalpiformis). Lendatus bolivianus can be distinguished from L. platys by the shape of the pronotum (becoming narrower (concave) posteriorly (Fig. 2A) in $L$.

bolivianus; becoming wide (convex) posteriorly (Fig. 2C) in L. platys); the shape of the paramere (paramere wider, converging to apex in dorsal view (Fig. 5B) in L. bolivianus; paramere narrower, parallel-sided from base to apex in dorsal view (Fig. 7B) in L. platys); and the length comparison between the anterior portion of the paramere and median lobe (median lobe slightly longer than paramere (Figs. 5A-B) in L. bolivianus; median lobe much longer than paramere (Figs. 7A-B) in L. platys).

Description. Forebody length 4.9-5.5 mm. Coloration of head, pronotum and ventral side of body dark brown to black; mouthparts and antennae dark orange; elytra metallic purple with green overtones; legs dark brown except tarsi dark orange; abdomen dark brown to black except segment 7 (posterior $1 / 4$ orange) and segment 8 (orange).

Peer) reviewing PDF | (2019:07:39356:1:1:NEW 16 Sep 2019) 
270

271

272

273

274

275

276

277

278

279

280

281

282

283

284

285

286

287

288

289

290

291

292

293

294

295

296

297

298

299

300

301

302

303

304

305

306

307

308

309

310

311

312

313

314

315

316

Head with 1-2 irregular rows of medium-sized punctures on each side of central impunctate area (except anteriorly); with additional 3-4 large punctures on epicranium; with microsculpture and micropunctures. Head width/length ratio $=1.61$. Pronotum width/length ratio $=0.95$; pronotum widest anteriorly, becoming gradually narrower posteriad; diagonal longitudinal line of punctures on disc of pronotum with 3-4 large punctures; anterolateral to that line pronotum with 5-6 medium-sized punctures; posterolateral to that line pronotum impunctate; pronotum with microsculpture and sparse micropunctures; pronotum/elytra length ration $=0.82$. Males with narrow, deep emargination on sternum 7; sternum 8 with deep Ushaped emargination.

Aedeagus as in Fig. 5; paramere in dorsal view gradually converging to rounded apex; in lateral view paramere slightly convex, converging to broadly rounded apex; paramere with peg setae as in Fig. 5C; paramere narrower but slightly longer than median lobe; median lobe in dorsal view converging to apex; in lateral view median lobe becoming narrower from middle to apex; with small dorsal subapical tooth.

Distribution. Known from the province of La Paz in Bolivia.

Habitat. All specimens were collected in the Yungas forest along eastern slope of the Andes Mountains in Bolivia (at elevations of $2000 \mathrm{~m}$ or above) by shifting litter or flight intercept traps.

Etymology. The specific epithet refers to the country of Bolivia.

\section{Lendatus philothalpiformis Chatzimanolis, new species}

(Figs. 1B, 2B, 3, 4, 6, 9)

urn:Isid:zoobank.org:act:7AFD3EE5-49B1-495D-A289-2C390B06BF61

Type material. Holotype, male, here designated, "Costa Rica: Puntarenas, Corcovado National Park, Sirena Station, upper Rio Claro trail, 100 m, 8'28'29' 'N 8335'8' 'W [8.474722, 83.58555], 28.Jun[e]-1.Jul[y].2000, Z.H. Falin, CR1ABF00 061, ex: flight intercept trap" / "SM0203906 [barcode label]" / "HOLOTYPE Lendatus philothalpiformis Chatzimanolis, des. Chatzimanolis 2019”. In the collection of SEMC.

Paratypes. 121: “Costa Rica: Alajuela, Estac. Biol. San Ramón, 900 m, 1.vii.-31.viii.1995, P. Hanson, CR1H93-95 5, ex: malaise trap" / "SM0075968" (1ð SEMC); same locality except 1.viii-30.ix.1995, CR1H93-95 6, SM0075818 (1 9 SEMC); same locality except 10¹3'4' 'N

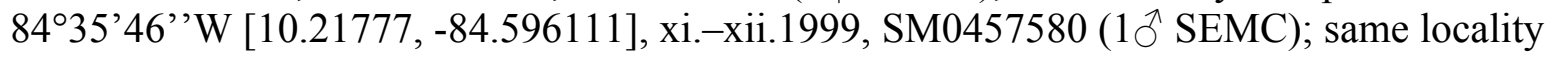
except 10¹3'4'’N 8435'46'’W [10.21777, -84.596111], ii.-iii.2000, SM0457607 (1 9 SEMC); “Costa Rica: Alajuela, E.B. San Ramón, R.B. San Ramón, 27 km N \& 8 km W San Ramón, 10¹3'30' 'N 8435'30' 'W [10.225, -84.591666], 850-950 m, 29.vi.-6.vii.1999, R. Anderson, wet premontane forest CR1A99-108A" / "SM0188194" (1ठ SEMC); same locality except 900 m, CR1A99-113B, SM0186510 (1 q SEMC); same locality except $810 \mathrm{~m}, 10^{\circ} 13$ '4' 'N 843' 46'’W [10.21777, -84.596111], 8.vii.2000, J.S. Ashe, R. Brooks, Z.H. Falin, CR1ABF00 084, ex: flight intercept trap, SM0203647, SM0203665 (1 $\jmath^{\lambda}, 1$ ㅇ SEMC); same locality except 900 m, 10¹3'4' 'N 84³5'46', W [10.21777, -84.596111], 8.vii.2000, P. Hanson, CR1EH99 01, SM0235433 (1ð SEMC); “Costa Rica: Prov. Alajuela, A.C.A. San Ramón, Reserva Biol Alberto

Peer) reviewing PDF | (2019:07:39356:1:1:NEW 16 Sep 2019) 
317 Brenes, Rio San Lorencito, 850 m, 24.iii.1999, C. Moraga, Sombrereta, L_N_245500_470800 318 \#52477” / INB0003030776, INB0003030777, INB0003030779 (2ð, 1 ○ NHMD); “Costa Rica: 319 Prov. Alajuela, San Ramón, Est. Biol. Villa Blanca, Send. La Capilla, 1115 m, 16.iii.-9.iv.2010, B. Hernández, Tp. Malaise, L_N_242482_483371 \#99630” / “INB0004248707” (1 q NHMD); "Costa Rica: Prov. Alajuela, Ūpala, P.N. Volcán Tenorio, Cerro La Carmela, 1026 m, 17.ii.18.iv.2010, J.A. Azofeifa, Tp. Malaise, L_N_298828_427338 \#99732” “INB0004256029” (1q NHMD); "Costa Rica: Alajuela, Peñas Blancas, 800 m, 19.v.1999, J.S. Ashe, R. Leschen, R. Brooks, ex: flight intercept trap" / "SM0046201" (1q SEMC); "Costa Rica: Prov. Alajuela, La Fortuna, Sector Catarata, 500 m, 3.xi.1997-6.i.1998, G. Garballo, Malaise, L_N_268500_462500 \#48837” / “INBIOCR002595077” (1 9 MNCR-A); “Costa Rica: Cartago Prov., Refugio Nac. de Fauna Silvestre Tapanti, 2 km E Station, 1320 m, 944.287’ N 8346.875'W [9.738116, -83.78125], 30.x.-1.xi.2001, R. Brooks, ex: flight intercept trap, CR1B01 15" / SM0474732, SM0474730, SM0474731, SM047429 (20̄, 1 q SEMC; $1 \overbrace{}^{\hat{~}} \mathrm{UTCI}$ ); same locality except $1 \mathrm{~km}$ E Station, $1410 \mathrm{~m}, 9^{\circ} 45.129^{\prime} \mathrm{N}$ 8346.936’ W [9.75215, -83.782266], CR1B01 13, SM0474724 (1 9 SEMC); “Costa Rica: Prov. Cartago, La Represa. Tapanti, 1800 m, vii.1995, R. Delgado, interseccion LN 185900563300 \#5342” / “INBIOCR002209951” (10 MNCR-A); "Costa Rica: Prov. Cartago, Pejibaye, Estación Biológica Copal, Sendero Tigra, 1090 m, 3-14.iv.2005, J. Azofeira Z., Tp. Malaise, L_N_196286_563684 \#80039” / "INB0003938486" (1 $\jmath^{\lambda}$ NHMD); "Costa Rica: Guanacaste, Guanacaste Conservacion Area, Maritza Field Station, 950 m, 13.ii.1996, R. Anderson, CR1A96 010C, ex: dry-tropical wet forest trans. litter" "SM0083887" (1О SEMC); "Costa Rica: Guanacaste, Estac. Cacao, 10001400 m, SW side Volcan Cacao, vii.1989-iii.1990, Malaise, TP.-GNP Biod. Survey” / INBIOCR000203134, INBIOCR000248458, INBIOCR000258332, INBIOCR000203124, INBIOCR000203105, INBIOCR000168862 (2今, 4ㅇ MNCR-A); same locality label except II curso Parataxon., vi.1990, INBIOCR000250397 (1 ڤ̂ MNCR-A); same locality label except iiiviii.1990, INBIOCR000231448 (1 9 MNCR-A); same locality label except 21-29.v.1992, INBIOCR000374813 (1 9 MNCR-A); same locality label except 1988-1989, INBIOCR000101546, INBIOCR000042128 (2q MNCR-A); “Costa Rica, Guanacaste, Estac. Pitilla, 9 km S Santa Cecilia, 700 m, xi.1989, C. Moraga \& P. Rios, 330200, 380200” / "INBIOCR000111406” (1 §̋ MNCR-A); "Costa Rica, Guanacaste, Tierras Morenas, 685 m, xi.1993, G. Rodriguez, L N 287800_427600 \#2476” / "INBIOCR001947013” (1 \& MNCR-A); "Costa Rica, Prov. Guanacaste, Macizo Miravalles, Estac. Cabro Muco. Sitio Azufral, 1100 m, 22.ix.-5.x.2003, J. Azofeifa, Intersección L_N_299769_411243 \#75479" / "INB0003771446" (19 NHMD); “Costa Rica: Heredia Prov., 6 km ENE Vara Blanca, $10^{\circ} 11^{\prime} \mathrm{N} 84^{\circ} 07^{\prime} \mathrm{W}$ [10.18333, -84.11666], 1950 m, 15-22.iv.2002, montane forest leaf litter, R. Anderson, CR2A02 03” / SM0527314, SM0527301 (2仓 SEMC); “Costa Rica, Heredia, Finca Murillo, 9 km NE Vara Blanca, 1450-1550 m, 10¹4'17' 'N 8406'06', W [10.238055, -84.101666], R. Anderson, 14-20.ii.2005, INbio-CET-ALAS transect, CRA105 007” / "SM0693946” (1 q SEMC); “Costa Rica: [Heredia] Vara Blanca, viii.[19]38" / "Field Mus. Nat. Hist.1966, A. Bierig Colln., Acc. Z13812” (1ठ FMNH); “Costa Rica: Prov. Limón, P.N. La Amistad. Punto., 1300-1400 m, 25.x.2.xi.2007, M. Moraga, B. Gamboa, Tp. Malaise, L_N_198990_627455 \#92615”/ "INB0004126042" (1 § NHMD); "Costa Rica: Prov. Limón, Manzanillo, RNFS Gandoca y Manzanillo, 0-100 m, 9.xi.-13.x.1992, K. Taylor, L-S 398100, 610600” / "INB000937676” (1우 MNCR-A); "Costa Rica: Puntarenas, Corcovado National Park, Sirena Station, Corcovado trail, $150 \mathrm{~m}, 8^{\circ} 29^{\prime} 7^{\prime}$ 'N 8334'39'’W [8.485277, -83.57750], 28.Jun[e]-1.Jul[y].2000, Z.H. Falin, CR1ABF00 059, ex: flight intercept trap" / "SM0203552” (1 $q$ SEMC); "Costa Rica: Puntarenas, 
363 Corcovado National Park, Sirena Station, Rio Pavo trail, $5 \mathrm{~m}, 8^{\circ} 29^{\prime} 5^{\prime}$ 'N 83 35'33' 'W

364 [8.484722, -83.5925], 25-28 Jun[e].2000, Z.H. Falin, CR1ABF00 037, ex: flight intercept trap" /

365 “SM0203763” (1ठ SEMC); "Costa Rica: Puntarenas, Monteverde, 24.v.1989, 1400 m, J.S.

366 Ashe, R. Leschen, R. Brooks, \#419, ex: pitfall trap" / "SM0046200” (1ð̊ SEMC); same locality

367 label except Boehme house, \#437, SM0046209 (1 $ð$ SEMC); same locality label except Cerro

368 Chomogo, $1550 \mathrm{~m}$, flight intercept trap, SM0046211 (1へ SEMC); same locality label except

$3691520 \mathrm{~m}$, flight intercept trap, SM0046199 (1ठึ SEMC); same locality label except $1570 \mathrm{~m}$,

370 9.v.1989, flight intercept trap, SM0046198 (1 \& SEMC); same locality label except $1630 \mathrm{~m}$,

371 7.vii.1990, S.E. Roberts, flight intercept trap, SM0046193 (1ð SEMC); same locality label

372 except 1610 m, 7.vii.1990, S.E. Roberts, flight intercept trap, SM0046210, SM0046208 (2ð

373 SEMC); same locality label except 21.v.1989, flight intercept trap, SM0046195, SM0046204,

374 SM0046202, SM0046197, SM0046194, SM0046205, SM0046196 (3ô, 2 q SEMC; $1 ð 1$ 우

375 UTCI); same locality label except $1550 \mathrm{~m}$, flight intercept trap, SM0046203 (1§ SEMC); same

376 locality label except 28-31.v.1992, M.L. Jameson, flight intercept trap, SM0045890 (1 ${ }^{\lambda}$

377 SEMC); “Costa Rica, Puntarenas, San Luis-Monteverde, LN250-850-449-250, 17-31.xii.1993,

378 Z. Fuentes, 1040 m, ex: malaise trap, \#2583” / "SM0068168” (1ठ SEMC); same locality label

379 except ii.1993, \#1897, SM0068198, INBIOCR002522864, INBIOCR002522865,

380 INBIOCR001166927 (1q SEMC; $3 q$ MNCR-A ); same locality label except ii.1992,

381 INBIOCR000842619 (1 9 MNCR-A); same locality label except 1000-1350 m, 17-31.xii.1992,

382 \#2583, INBIOCR002523162 (1へ MNCR-A); same locality label except 1000-1350 m, xii.1993,

383 \#2493, INBIOCR001714070 (1○ึ MNCR-A); same locality label except vii.1993, \#2424,

384 INBIOCR002523005 (1ठ MNCR-A); same locality label except 1-31.x.1993, \#2425,

385 INBIOCR001957088 (1ð̋ MNCR-A); same locality label except vii.1992, INBIOCR000722993

386 (1ð MNCR-A); same locality label except x.1993, \#2428, INBIOCR002523051 (1ð MNCR-A);

387

388

389

390

391

same locality label except xi.1993, \#2443, INBIOCR001938006, INBIOCR001938032,

INBIOCR001938005, INBIOCR001938033 (20, 2 \% MNCR-A); same locality label except

ix.1993, \#2429, INBIOCR002523059 (1ð̋ MNCR-A); same locality label, A. C. Arenal, xi.1993, \#2427, Z. Fuentes, Amarilla, SM0068204, SM0068201, INBIOCR002523429,

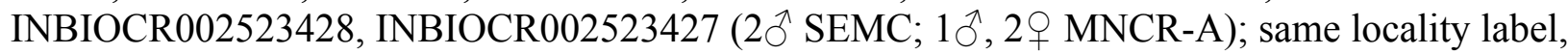

392

393

394

395

396

397

398

A. C. Arenal, i.1993, Z. Fuentes, LN 449250_250850 \#2584, SM0068203, INBIOCR002523178,

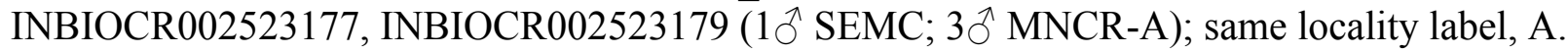

C. Arenal, i.1993, Z. Fuentes, LN 449250_250850 \#2585, SM0068196, SM0068200 (2ㅇ

SEMC); same locality label except 20-27.vi.1994, \#3029, INBIOCR001922841,

INBIOCR001922842 (1ð̄, 1 q MNCR-A); “Costa Rica, Puntarenas, Res. Biol. Monteverde, Est.

La Casona, 1520 m, K. Flores, iv.1992, L-N 253250 449700” / INBIOCR000990559,

INBIOCR000793519 (1ڤ̂, 1 q MNCR-A); same locality label except ix.1991,

399

400

401

402

403

404

405

406

407

408

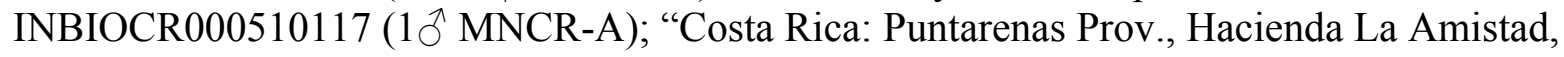
$8^{\circ} 58.102^{\prime} \mathrm{N} 82^{\circ} 46.883$ ’W [8.968366,-82.781383], $1900 \mathrm{~m}$, premont.-lower mont. moist forest, sifting leaf litter, 12.vi.2012, Solodovnikov, Brunke, Puliafico, Selvantharan" / "Chatzimanolis DNA Voucher, Extraction SC-405, Extraction date: 27.iii.2015” (1ठ NHMD); “Costa Rica, Puntarenas, R.F. Golfo Dulce, 3 km SW Rincon, 10 m, v-vi.1992, P. Hanson, ex: malaise” / "SM0069525" (1 $\delta^{\Uparrow}$ SEMC); "Costa Rica, Puntarenas, Altamira Biol. Sta. 1510-1600 m, 9¹.76’N 830.49’W [9.029333, -83.008166], 4-7.vi.2004, J. S. Ashe, Z.H. Falin, I. Hinojosa, ex: flight intercept trap, CR1AFH04 144” / “SM0606679” (1 § SEMC); "Costa Rica, Puntarenas, Las Alturas Biol. Sta. 1660 m, 856.17’N 8250.01’W [8.936166, -82.8335], 31.v.-3.vi.2004, J. S. Ashe, Z.H. Falin, I. Hinojosa, ex: flight intercept trap, CR1AFH04 092" / "SM0606867” (1웅

Peer) reviewing PDF | (2019:07:39356:1:1:NEW 16 Sep 2019) 
409 SEMC); “Costa Rica: Puntarenas, Fca. Cafrosa, Est. Las Mellizas, P.N. Amistad., 1300 m, R. 410 Delgado, 19.vi.-26.vii.1990, L-S-316100, 596100" / "INBIOCR000667816” (1ð MNCR-A); "Costa Rica, San Jose, Zurqui de Moravia, 1600 m, iv.1994, P. Hanson, ex: malaise" / "SM0069535" (1ठ SEMC); same locality except iii.1994, SM0069520 (1ð SEMC); same locality except 1-30.viii.1995, CR1H93-95 14, SM0077306 (1ठ SEMC); same locality except 10³'0'’ $\mathrm{N} 84^{\circ} 1^{\prime} 0$ '’ W [10.05, -84.01666], 1-30.ix.1995, CR1H95-96 07, SM0134461 (1へ SEMC); "Costa Rica: San Vito de C. B., Las Cruces, 1200 m, 9.vii.-7.viii.1982, malaise tr, B. Gill” (1 9 CNC); "Panama: Bocas del Toro, 4 km N. Boquete, La Culebra trail, 1500 m,

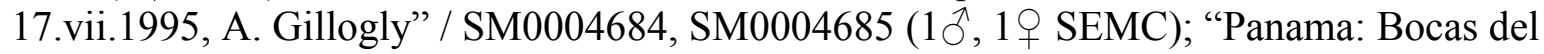
Toro, $8^{\circ} 34 \mathrm{~N}$ 81 ${ }^{\circ} 50 \mathrm{~W}$ [8.56666, -81.83333], 1500 m, 25 km NNE San Felix, leg. J. Wagner, 6.vi.1980" / "FM(HD)\#80-5, Berlese floor litter \& root mat, nr. ridge top, Qda. Alicia cloud forest" (1 9 FMNH); same locality except 10-12.vi.1980, Camino I7, malaise trap (1 9 FMNH); “Panama: Chiriqui Prov., La Fortuna Cont. Divide Trail, 846’N 82¹2’W [8.766666, -82.2], 1150 m, 23.v.-9.vi.1995, J. Ashe, R. Brooks, \#155, ex: flight intercept trap" / SM0046213, SM0007044, SM0003729, SM0046214 (3ð̄, 1ㅇ SEMC); same locality except 1100 m, \#157, SM0003687 (1 $q$ SEMC); same locality except, 9-12.vi.1995, \#185, SM0003498 (1 9 SEMC); same locality except $1200 \mathrm{~m}$, 9.vii.1995, R. Anderson, PAN2A95 10C, ex: berlese forest litter, SM0037220 (1 9 SEMC); same locality except Hydrolog. Trail, 842’ N 82 ${ }^{\circ} 14^{\prime} \mathrm{W}, 1050 \mathrm{~m}, 9$ 12.vi.1995, \#188, SM0005052 (1 ð` SEMC); same locality Hydrolog. Trail, $8^{\circ} 42^{\prime} \mathrm{N} 82^{\circ} 14^{\prime} \mathrm{W}$ [8.7, -82.233], 1150 m, 23.v.-9.vi.1995, \#156, SM0003747 (1 9 SEMC); "Panama: Chiriqui, 4 km N Sta. Clara Hartmann's Finca, 27.vi.-3.vii.1981, B. Gill, 1500 m” (1 + CMNC); "Panama: Chiriqui, La Fortuna Dam, 1200 m, 14.vi.-15.viii.1982, wet forest FIT, B. Gill” (1 9 CNC); All paratypes with label "PARATYPE Lendatus philothalpiformis Chatzimanolis, des. Chatzimanolis 2019".

Diagnosis. Lendatus philothalpiformis can be easily recognized among the existing species in the genus due to the bright reddish-orange coloration of the head and pronotum. Additionally, it is the only species known with a Central American distribution.

Description. Forebody length 4.6-5.8 $\mathrm{mm}$. Coloration of head, pronotum and prosternum bright reddish-orange (in few specimens brown); mouthparts, antennae and legs reddish-orange to brown; elytra metallic green or blue; meso- and metaventrite brown; abdomen reddish-orange to brown (frequently with segment 6 dark brown) except segment 7 dark brown with posterior 1/3 orange and segment 8 orange.

Head with 1-2 irregular rows of large punctures on each side of central impunctate area (except anteriorly); with additional 3-4 large punctures on epicranium; with microsculpture and micropunctures. Head width/length ratio $=1.5$. Pronotum width/length $\mathrm{ratio}=0.92$; pronotum widest anteriorly, becoming strongly narrower (concave) posteriad; diagonal longitudinal line of punctures on disc of pronotum with 3-4 large punctures; anterolateral to that line pronotum with less than 5 large punctures; posterolateral to that line pronotum impunctate; pronotum with microsculpture and micropunctures; pronotum/elytra length ration $=0.92$. Males with broad, shallow margination on sternum 7 (Fig. 4C); sternum 8 with shallow V-shaped emargination.

Aedeagus as in Fig. 6; paramere in dorsal view almost parallel-sided but apex wider; in lateral view paramere slightly convex, converging to narrow rounded apex; paramere with peg setae as in Fig. 6C; paramere narrower than median lobe except just before apex; paramere longer than median lobe; median lobe in dorsal view converging to apex; in lateral view median 
455 lobe becoming narrower from middle to narrowly elongate apex; with large dorsal subapical 456 tooth.

457

458

459

460

461

462

463

464

465

466

467

468

469

470

471

472

473

474

475

476

477

478

479

480

481

482

483

484

485

486

487

488

489

490

491

492

493

494

495

496

497

498

499

500

501

Distribution. Known from many provinces in Costa Rica and the provinces of Bocas del Toro and Chiriqui in Panama.

Habitat. Specimens were collected with malaise, pitfall and flight intercept traps and by shifting leaf litter in wet tropical lowland forests or tropical cloud forests at elevations of 5-1950 m).

Etymology. The specific epithet is derived from the words Philothalpus and formis and refers to the superficial resemblance of this species to species in the genus Philothalpus.

\section{Lendatus platys Chatzimanolis, new species}

(Figs. 1C, 2C, 7, 8)

urn:Isid:zoobank.org:act:920D79A5-D1D4-4B73-8263-30D4908E3823

Type material. Holotype, here designated, male, "Ecuador, Sucumbios, Sacha Lodge, $0.5^{\circ} \mathrm{S}$ 76.5 $\mathrm{W}$ [-0.5, -76.5], $270 \mathrm{~m}, 13-23 . v i .1994$, Hibbs, ex: malaise" / "SM0022600 [barcode label]" / "HOLOTYPE Lendatus platys Chatzimanolis, des. Chatzimanolis 2019". In the collection of SEMC.

Paratypes. 48: same locality label as holotype, SM0022371 (1ð SEMC); same locality label as holotype except 14-24.v.1994, SM0023298 (1 P SEMC); same locality label as holotype except 3-16.viii.1994, SM0020931 (1 오 SEMC); "Bolivia: Santa Cruz, Amboro National Park, Los Volcanes, c. $1000 \mathrm{~m}, 18^{\circ} 06^{\prime} \mathrm{S}$ 633' $\mathrm{W}$ [-18.1, -63.6], 20.xi.-12.xii.2004" / "flight interception trap, H. Mendel \& M.V.L. Barclay, BMNH(E) 2004-280" (50, 2 q BMNH); "Ecuador: MoronaSantiago, Macas, 1300 m, 20.ix.1989, M. Cooper" / "M. Cooper BMNH(E) 2004-275" (1ㅇ BMNH); "Ecuador: Napo, Yuturi Lodge, Rio Napo, 270 m, 0 $0^{\circ} 32^{\prime} 54^{\prime}$ 'S 76²'18' 'W [-0.548333, -76.03833], 20-21.iii.1999, R. Brooks, D. Brzoska, ECU1B99 010, ex: flight intercept trap" / SM0153450, SM0153439, SM0153432, SM0153459 (3ð SEMC; 1ð UTCI); "Ecuador: Napo,

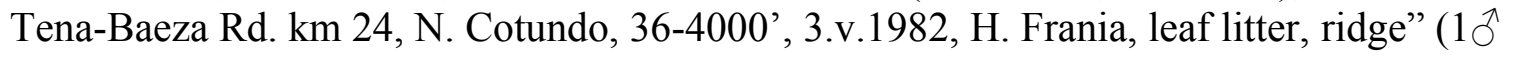
FMNH); “Ecuador, Napo Prov. Yasuni N.P., Yasuni Research Sta., $0^{\circ} 38^{\prime}$ 'S 76³6’W [-0.6333, 76.6], 215 m, 27.vii.-1.viii.1998, lowland rainforest, Ratcliffe, Jameson, Smith, Villatoro (10 UNSM); “Ecuador: Napo, Yasuni Nat. Park Biol. Res. Station, $220 \mathrm{~m}, 0.67^{\circ} \mathrm{S} 76.39^{\circ} \mathrm{W}$ [-0.67, 76.39], 18-26.v.1996, P. Hibbs, MT, primary forest (1 9 CNC); "Ecuador: Prov. Orellano, Yasuni Natl. Park, Yasuni Research Stn., $0^{\circ} 40^{\prime} 50^{\prime}$ 'S 76 24'2' 'W [-0.680555, -76.400555], 250 m, 28.iv.-8.v.2009, on lead, M. Cannon (1 $\overbrace{}^{\Uparrow}$ DEBU); "Ecuador: Sucumbios, Sacha Lodge, 270 m, 0'28'14'’S 76²7'35' 'W [-0.470555, -76.45972], 21-24.iii.1999, R. Brooks, ECU1B99 047, ex: flight intercept trap" / SM0153262, SM0153263, SM0153255 (3ð SEMC); "Ecuador: [Sucumbios], Napo R. Sacha Lodge, 250 m, 26-28.x.2004, FIT, G. de Rougemont leg. (1우

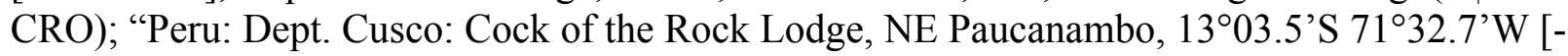
13.05833, -71.545], $1120 \mathrm{~m}, 4-9 . x i .2007$, D. Brzoska, ex. flight intercept trap, PER1B07 001"/

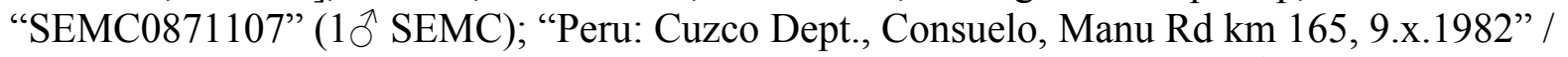

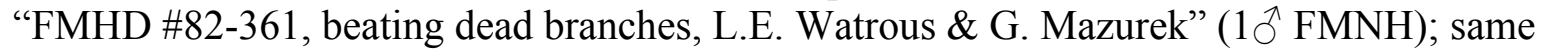
locality labels except 4.x.1982, FMHD \#82-337, leaf litter (1 9 FMNH); same locality labels 
502 except 5.x.1982, FMHD \#82-410, rotten palm bait trap (1§ FMNH); same locality labels except 503 6-7.x.1982, FMHD \#82-411, flight intercept trap (1§ FMNH); same locality labels except 504 Pillahuata, Manu Rd. km 128, 20.ix.1982, FMHD \#82-265, litter along gravel stream (1 $\sigma^{\lambda}$ 505 FMNH); same locality labels except Pillahuata, Manu Rd. km 128, 24.ix.1982, FMHD \#82-283,

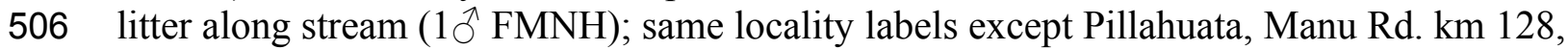
507 27.ix.1982, FMHD \#82-310, litter in runoff in mossy forest (1§, 19 FMNH); same locality 508 labels except Pillahuata, Manu Rd. km 128, 28.ix.1982, FMHD \#82-311, litter along gravel stream (2 $\overbrace{}^{\lambda} \mathrm{FMNH})$; “Peru: CU[sco] Camparmento Comerciato, 23.xi.2002, $12^{\circ} 47^{\prime} \mathrm{S} 73^{\circ} 22^{\prime} \mathrm{W}$ [12.78333, -73.36666], 1350 m, Pitfall, J. Grados" / “Isanopus spp. det. Asenjo 2004” (1ठ MUSM); "Peru: CU[sco] Camparmento Segakiato, 10.xi.2002, 1243'S 7318'W [-12.716666, 73.3], 1850 m, Pitfall, J. Grados" (1甲 MUSM); "Peru: Dept. Loreto, Camparmento San Jacinto, $2^{\circ} 18.75^{\prime}$ S 75'51.77W [-2.3125, -75.862833], 7.vii.1993, 175-215 m, R. Leschen \#44, ex: flight intercept trap" / "SM0080093" (1ㅇ SEMC); "Peru: Dept. Loreto, 1.5 km N. Teniente Lopez, $2^{\circ} 35.66^{\prime} \mathrm{S} 76^{\circ} 06.92$ 'W [-2.594333, -76.115333], 18.vii.1993, 210-240 m, R. Leschen, \#119. ex: flight intercept trap" / "SM0080094” (1 9 SEMC); "Peru: JU[nín], 1 km S Mina Pichita, 2100 m,

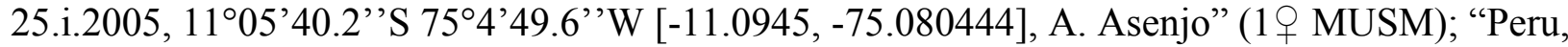
Dept. Madre de Dios: Pantiacolla Lodge, Alto Madre de Dios R., 12³9.3'S 71¹3.9W [-12.655, -71.231666], 420 m, 14-19.xi.2007, D. Brzoska, ex. flight intercept trap, PER1B07 004” / "SEMC0872413” (1 § SEMC); "Peru: Madre de Dios: Pantiacolla Lodge, 8 km NW El Mirador Trail, Alto Madre de Dios River, 800 m, 12³8'30''S 71¹6'41'’W [-12.64166, -71.278055], 23-26.x.2000, R. Brooks, PERU1B00 102, ex: flight intercept trap" / SM0210891, SM0210653

523

524

525

526

527 (1 $\odot$ SEMC; 1 UTCI); "Peru, Madre de Dios Dept., CICRA Field Station, trail 6, research plot, $12.55207^{\circ} \mathrm{S} 70.10962^{\circ} \mathrm{W}, 295 \mathrm{~m}, 11-13 . v i .2011$, Chaboo team, Malaise trap, PER-11-MAT021" / "SEMC1060728” (1ठ̋ SEMC); "Peru, Madre de Dios Dept., CICRA Field Station, 2 km $\mathrm{NW}$ of cafeteria, research plot, $12.55212^{\circ} \mathrm{S} 70.10921^{\circ} \mathrm{W}, 295 \mathrm{~m}, 7-9 . v i .2011$, Chaboo team,

528

529

530 flight intercept trap trap, PER-11-FIT-021" / "SEMC0956719” (1 $q$ SEMC); "Peru: Dept. Madre de Dios, Manu Prov., Parque Nac. Manu, Zona Res. Rio Manu, Cocha Juarez, trail nr. Manu" /

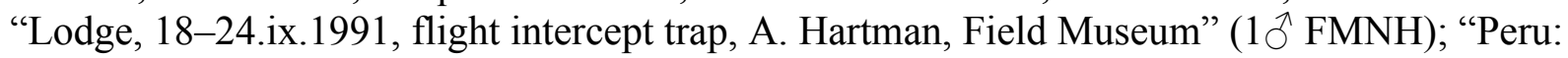
Madre de Dios, Tambopata Wildlife Res. 30 km SW Pto. Maldanado, 1250’S 69²0’W [12.83333, -69.3333], 290 m, 26.xi.1982, J.J. Anderson coll.” (1 $\left.\bigcirc^{\Uparrow} \mathrm{CMNH}\right)$; "Peru, Ucauali Dept., Tingo Maria-Pucallpa Rd., Ruente Chino, km 205, 1300 m, 98'12' 'S 7547'20' 'W [-9.13666, 75.788888], 11-14.x.1999, R. Brooks, PERU1B99 007A, ex: flight intercept trap" / SM0185071, SM0185076 (1ठึ SEMC; $1 \hat{\jmath}$ UTCI). All paratypes with label "PARATYPE Lendatus platys Chatzimanolis, des. Chatzimanolis 2019".

Diagnosis. Lendatus platys and L. bolivianus can be distinguished from L. philothalpiformis by the coloration of head and pronotum (dark brown to black in L. bolivianus and L. platys; bright reddish-orange in L. philothalpiformis). Lendatus platys can be distinguished from L. bolivianus

540

541

542

543

544 by the shape of the pronotum (becoming wide (convex) posteriorly (Fig. 2C) in L. platys; becoming narrower (concave) posteriorly (Fig. 2A) in L. bolivianus;); the shape of the paramere (paramere narrower, parallel-sided from base to apex in dorsal view (Fig. 7B) in L. platys; paramere wider, converging to apex in dorsal view (Fig. 5B) in L. bolivianus;) and the length comparison between the anterior portion of the paramere and median lobe (median lobe much longer than paramere (Figs. 7A-B) in L. platys; median lobe slightly longer than paramere (Figs. 5A-B) in L. bolivianus). 
548 Description. Forebody length 4.7-5.6 mm. Coloration of head, pronotum and ventral side of 549 body dark brown to black; mouthparts and antennae dark orange to brown; elytra metallic blue, 550 green or purple (blue most commonly); legs dark brown except tarsi dark orange; abdomen dark

551

552

553

554

555

556

557

558

559

560

561

562

563

564

565

566

567

568

569

570

571

572

573

574

575

576

577

578

579

580

581

582

583

584

585

586

587

588

589

590

591

brown to black except segment 7 (posterior $1 / 3$ orange) and segment 8 (orange).

Head with 2-3 irregular rows of medium-sized punctures on each side of central impunctate area (except anteriorly); with additional 4-6 large punctures on epicranium; with microsculpture and micropunctures. Head width/length ratio $=1.53$. Pronotum width/length ratio $=1.02$; pronotum widest medially, lateral sides of pronotum convex; diagonal longitudinal line of punctures on disc of pronotum with 5-6 large punctures; anterolateral to that line pronotum with 5-8 medium-sized punctures; posterolateral to that line pronotum impunctate; pronotum with microsculpture and micropunctures; pronotum/elytra length ration $=0.89$. Males with narrow, deep emargination on sternum 7; sternum 8 with deep U-shaped emargination.

Aedeagus as in Fig. 7; paramere in dorsal view almost parallel-sided but apex slightly wider; in lateral view paramere convex, converging to broadly rounded apex; paramere with peg setae as in Fig. 7C; paramere narrower but longer than median lobe; median lobe in dorsal view converging to apex; in lateral view median lobe becoming narrower from middle to narrowly elongate apex; with small dorsal subapical tooth.

Distribution. Known from the department of Santa Cruz in Bolivia, the provinces of MoronaSantiago, Napo, Orellano and Sucumbios in Ecuador, and the departments of Cusco, Loreto, Junín, Madre de Dions and Ucauali in Peru.

Habitat. Specimens were collected with malaise, baited pitfall and flight intercept traps and by shifting leaf litter in wet tropical lowland forests or tropical cloud forests at elevations of 10 $1300 \mathrm{~m})$.

Etymology. The specific epithet is derived from the Greek word $\pi \lambda \alpha \tau$ c (wide) and refers to the wide shape of the pronotum.

Remarks. An additional specimen from Colombia [Colombia: Valle del Cauca, PNN Farallones de Cali, Anchicaya, $3^{\circ} 26^{\prime} \mathrm{N} 76^{\circ} 48^{\prime} \mathrm{W}, 730 \mathrm{~m}$, 27.ii.-27.iii.2001, Malaise, S. Sarria leg., M1538”' / "SM0548730"(1우 SEMC)] looks almost identical to this species, except that the pronotum is not as wide as the other specimens in this species. Unfortunately, this specimen is female and thus I cannot place it with certainty in L. platys.

\section{Key to the species of Lendatus}

1. Color of head and pronotum (Fig. 2B) bright reddish-orange (rarely brown); distributed in Central America (Fig. 9) ... Lendatus philothalpiformis

- $\quad$ Color of head and pronotum dark brown to black (Figs. 2A, C); distributed in South America (Fig. 8) ............................... 2 
592 2. Pronotum becoming narrower (concave) posteriorly (Fig. 2A); paramere wider, 593 converging to apex in dorsal view (Fig. 5B); anterior portion of median lobe slightly longer than

594

595

596

597

598

599

600

601

602

603

604

605

606

607

608

609

610

611

612

613

614

615

616

617

618

619

620

621

622

623

624

625

626

627

628

629

630

631

632

633

634

635

636 paramere (Figs. 5A-B) ... Lendatus bolivianus

- $\quad$ Pronotum becoming wide (convex) posteriorly (Fig. 2C); paramere narrower, parallelsided from base to apex in dorsal view (Fig. 7B); anterior portion of median lobe much longer than paramere (Figs. 7A-B) ... Lendatus platys

\section{Acknowledgements}

I thank all the curators and collection managers listed in the materials examined section for access to the specimens. I thank Adam Brunke, Aslak Kappel Hansen and an anonymous reviewer for comments that improved this manuscript. I thank Max Marlowe for taking some of the photographs shown in this paper.

\section{References}

Chani-Posse, M.R., Brunke, A.J., Chatzimanolis, S., Schillhammer, H., Solodovnikov, A. (2018) Phylogeny of the hyper-diverse rove beetle subtribe Philonthina with implications for classification of the tribe Staphylinini (Coleoptera: Staphylinidae). Cladistics, 38, 1-40. https://doi.org/10.1111/cla.12188

Chatzimanolis, S. (2008) A revision of the neotropical beetle genus Isanopus (Coleoptera:

Staphylinidae: Staphylinini). Journal of Natural History, 42 (25-28), 1765-1792. https://doi.org/10.1080/00222930802124057

Chatzimanolis, S. (2012) Zackfalinus, a new genus of Xanthopygina (Coleoptera: Staphylinidae: Staphylinini) with description of 20 new species. Annals of the Carnegie Museum, 80, 261-308. http://dx.doi.org/10.2992/007.080.0401

Chatzimanolis, S. (2014) Phylogeny of xanthopygine rove beetles based on six molecular loci. Systematic Entomology, 39 (1), 141-149.

https://doi.org/10.1111/syen.12040

Chatzimanolis, S \& Brunke, A.J. (2019) A phylogeny of Xanthopygina (Insecta: Coleoptera) reveals major lineages and the origin of myrmecophily. Zoologica Scripta, 48, 494-506. https://doi.org/10.1111/zsc.12358

Chatzimanolis, S \& Hightower, H.J. (2019) Peripus, a new genus of Xanthopygina (Coleoptera: Staphylinidae) from South America. Zootaxa, 4648 (2), 371-383.

https://doi.org/10.11646/zootaxa.4648.2.10 
637

638 Shorthouse, D.P. (2010) SimpleMappr, an online tool to produce publication-quality point maps.

639

640

641 Wheeler, Q.D. \& Platnick. N.I. (2000) The phylogenetic species concept (sensu Wheeler and

642 Platnick). In: Q.D. Wheeler \& Meier, R. (Eds.), Species Concepts and Phylogenetic Theory: A

643 Debate). Columbia University Press, New York, NY, pp. 55-69.

644

645

646 
Figure 1

Habitus photographs of species of Lendatus Chatzimanolis.

(A) Lendatus bolivianus Chatzimanolis. (B) Lendatus philothalpiformis Chatzimanolis. (C) Lendatus platys Chatzimanolis. Not to scale.

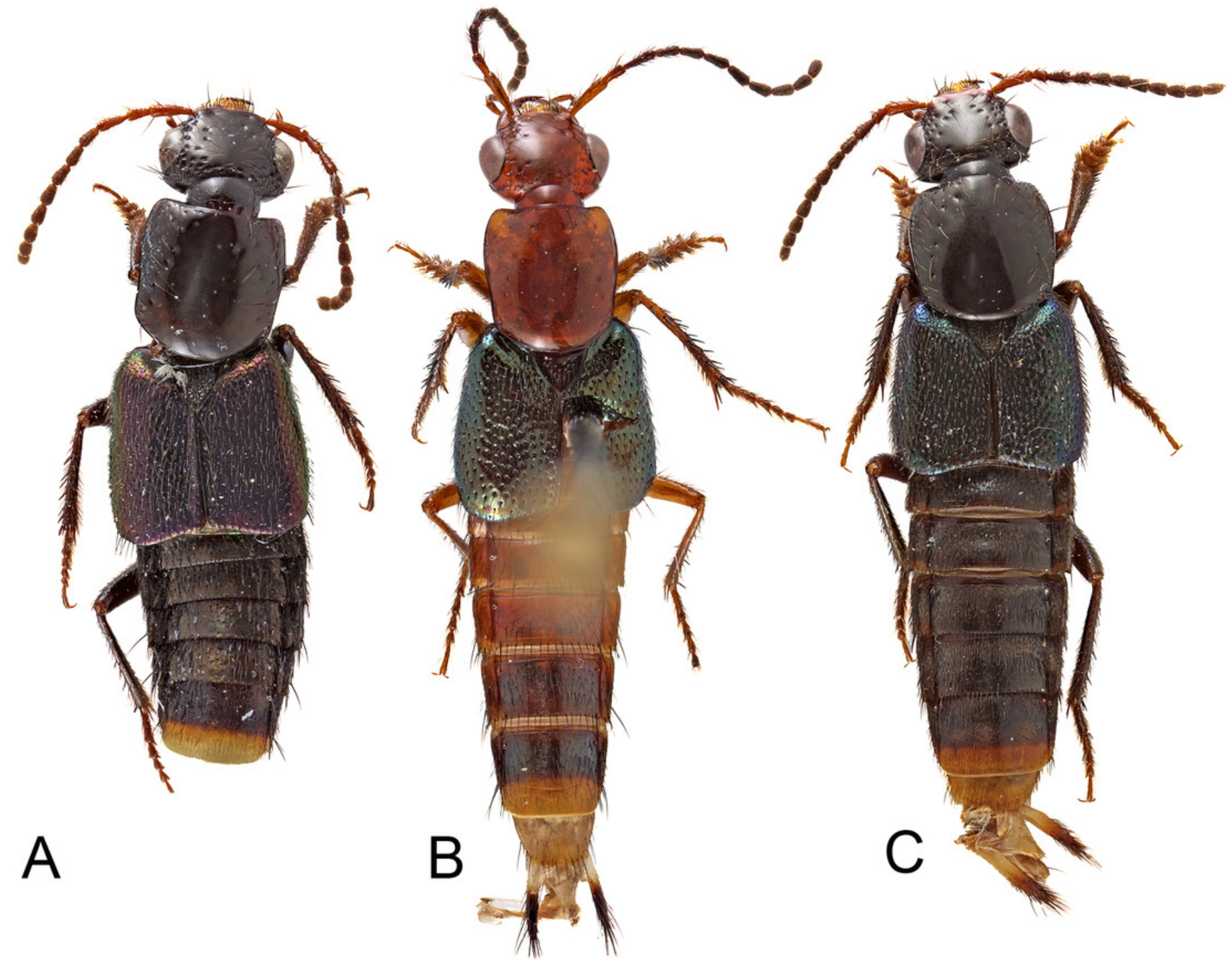




\section{Figure 2}

Heads and pronota of species of Lendatus Chatzimanolis.

(A) Lendatus bolivianus Chatzimanolis. (B) Lendatus philothalpiformis Chatzimanolis. (C) Lendatus platys Chatzimanolis. Not to scale.

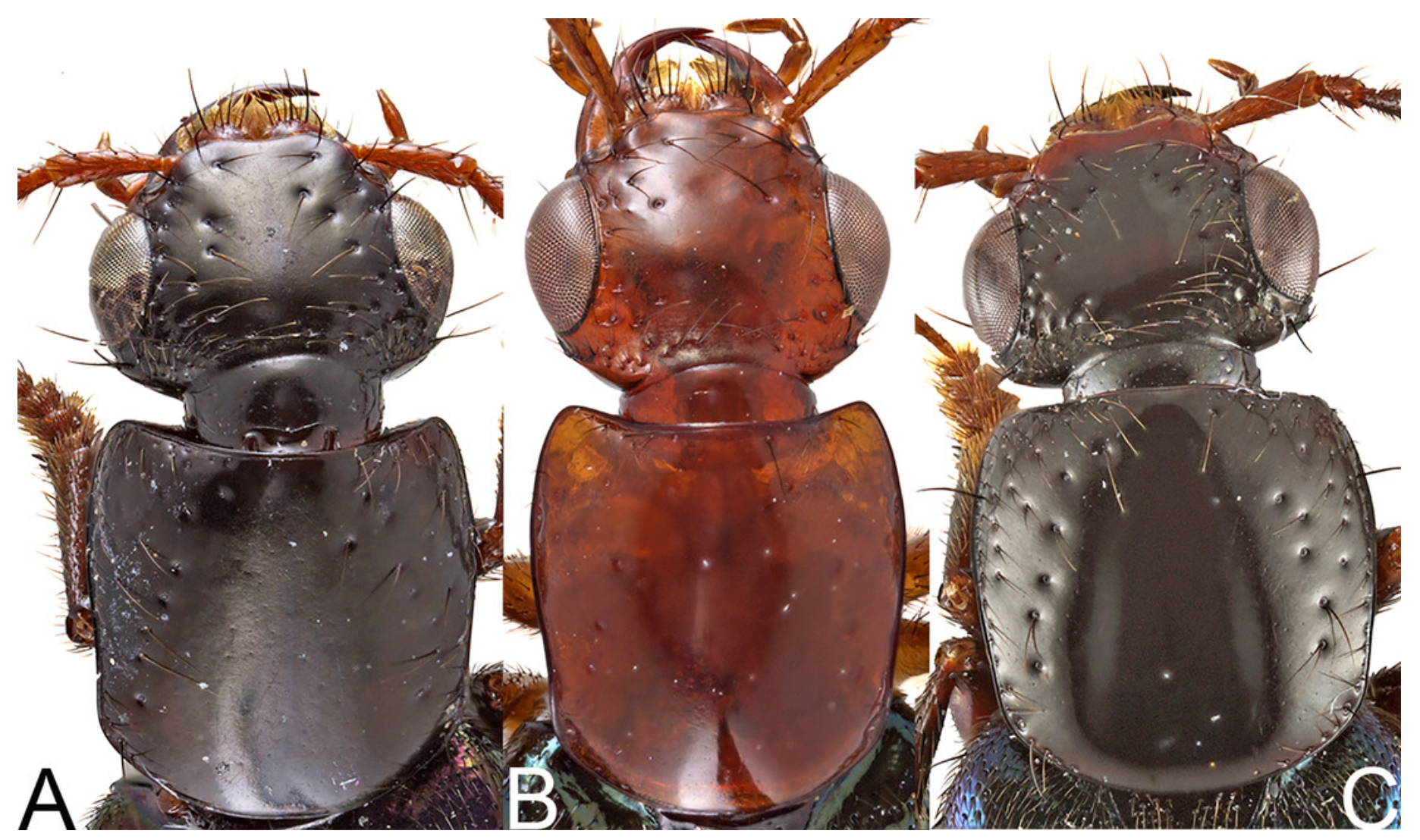


Figure 3

SEM photographs of Lendatus philothalpiformis Chatzimanolis.

(A) Ventral view of left mandible, scale bar $=0.56 \mathrm{~mm}$. (B) Dorsal view of right mandible, scale bar $=0.56 \mathrm{~mm}$. (C) Hypopharynx and labial palps, scale bar $=0.88 \mathrm{~mm}$. (D) Maxilla, scale bar $=0.56 \mathrm{~mm}$. (E) Antenna, scale bar $=1.09 \mathrm{~mm}$. Numbers above the antenna, the maxillary palp and the labial palp correspond to the different segments; ga: galea; Ic: lacinia; hp: hypopharynx. 


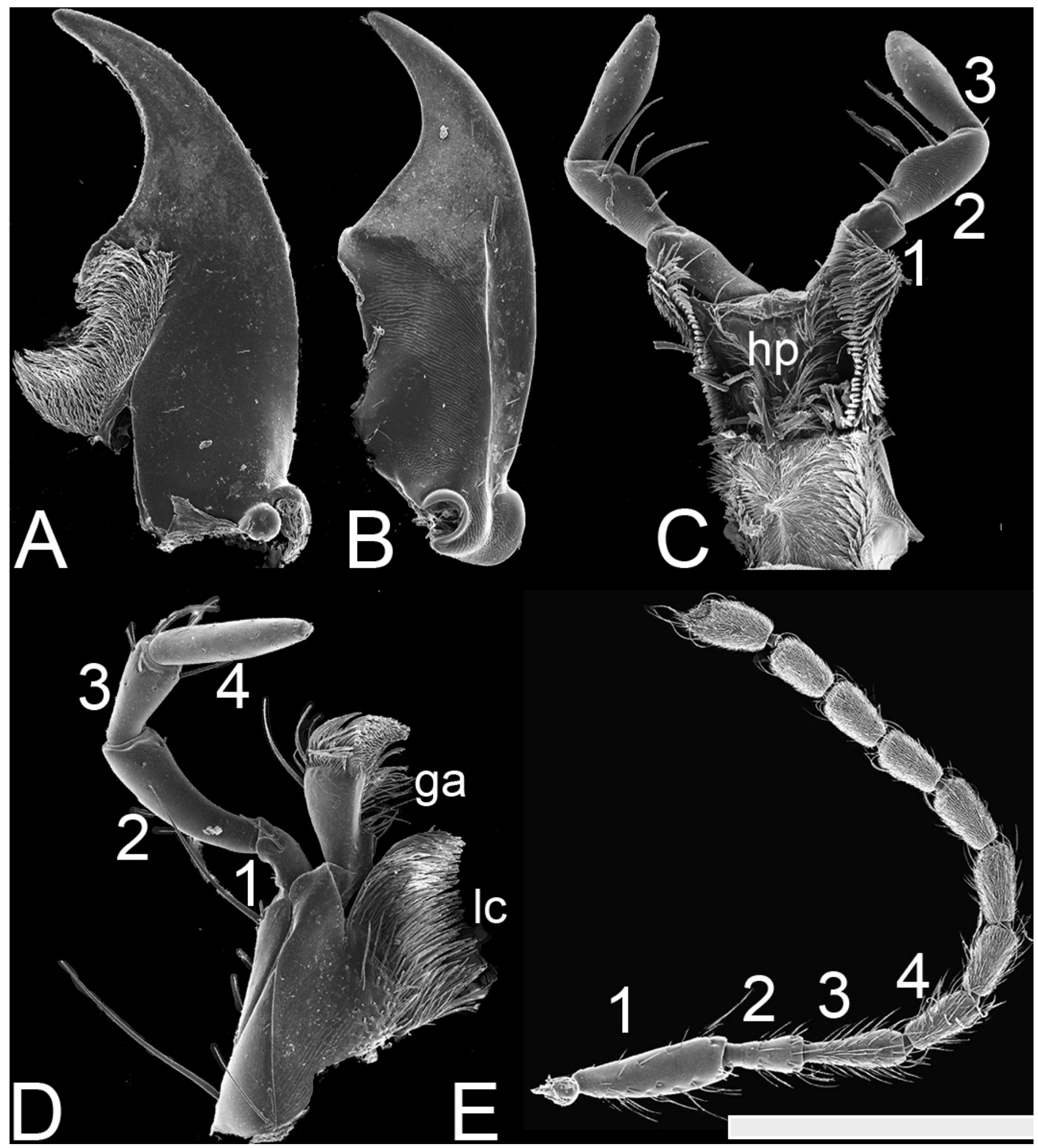


Figure 4

SEM photographs of Lendatus philothalpiformis Chatzimanolis.

(A) Prosternum and pronotal hypomeron, scale bar $=1.09 \mathrm{~mm}$. (B) Meso- and metaventrite, scale bar $=1.44 \mathrm{~mm}$. (C) Abdominal sterna 5-7, scale bar $=1.25 \mathrm{~mm}$. (D) Abdominal sterna $8-9$, scale bar $=1.27 \mathrm{~mm}$. Numbers on the abdomen correspond to the number of segments; ms: mesoventrite; mt: metaventrite. 


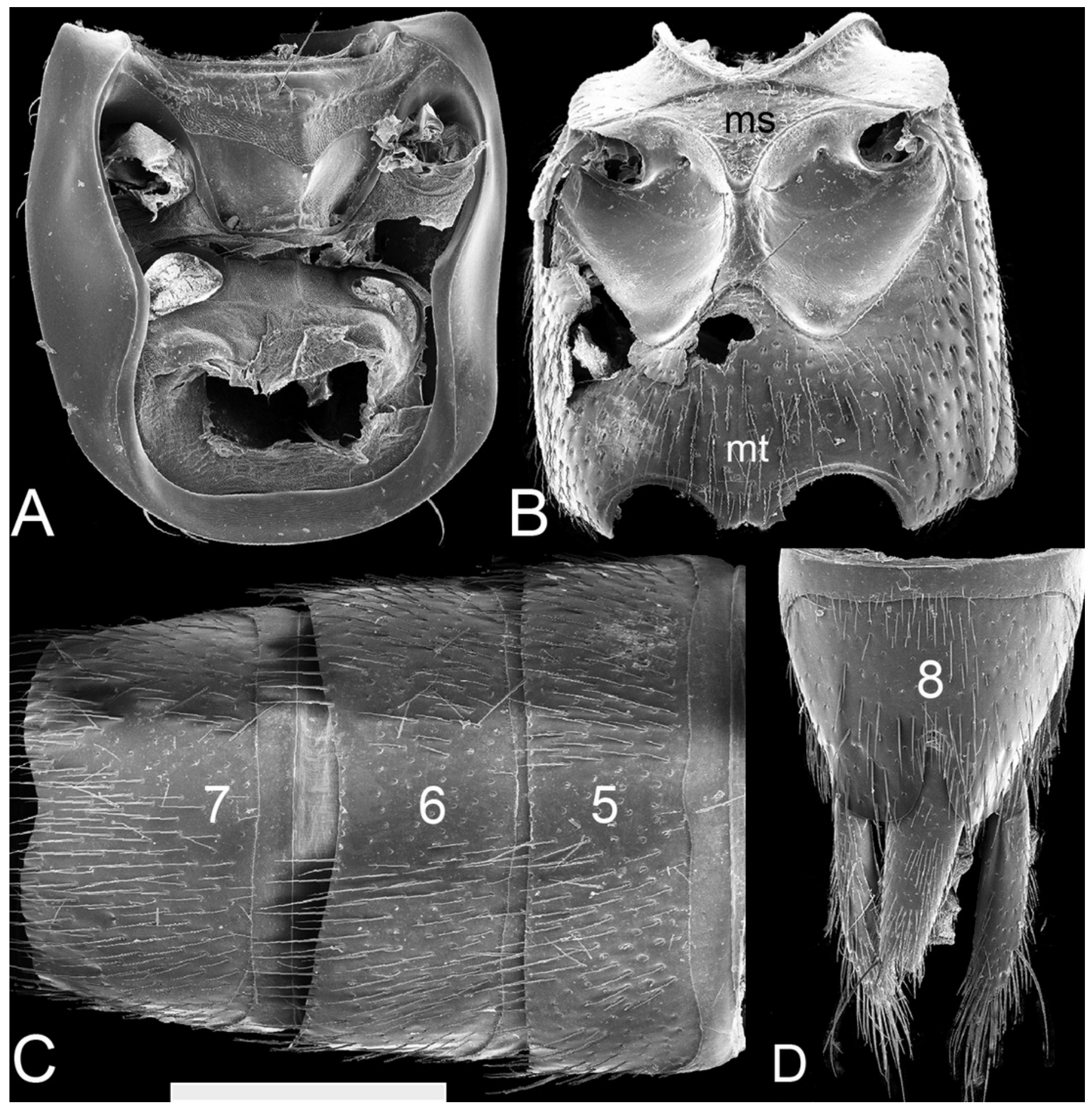


Figure 5

Aedeagus of Lendatus bolivianus Chatzimanolis.

(A) Lateral view. (B) Dorsal view. (C) Detail of paramere, ventral view.
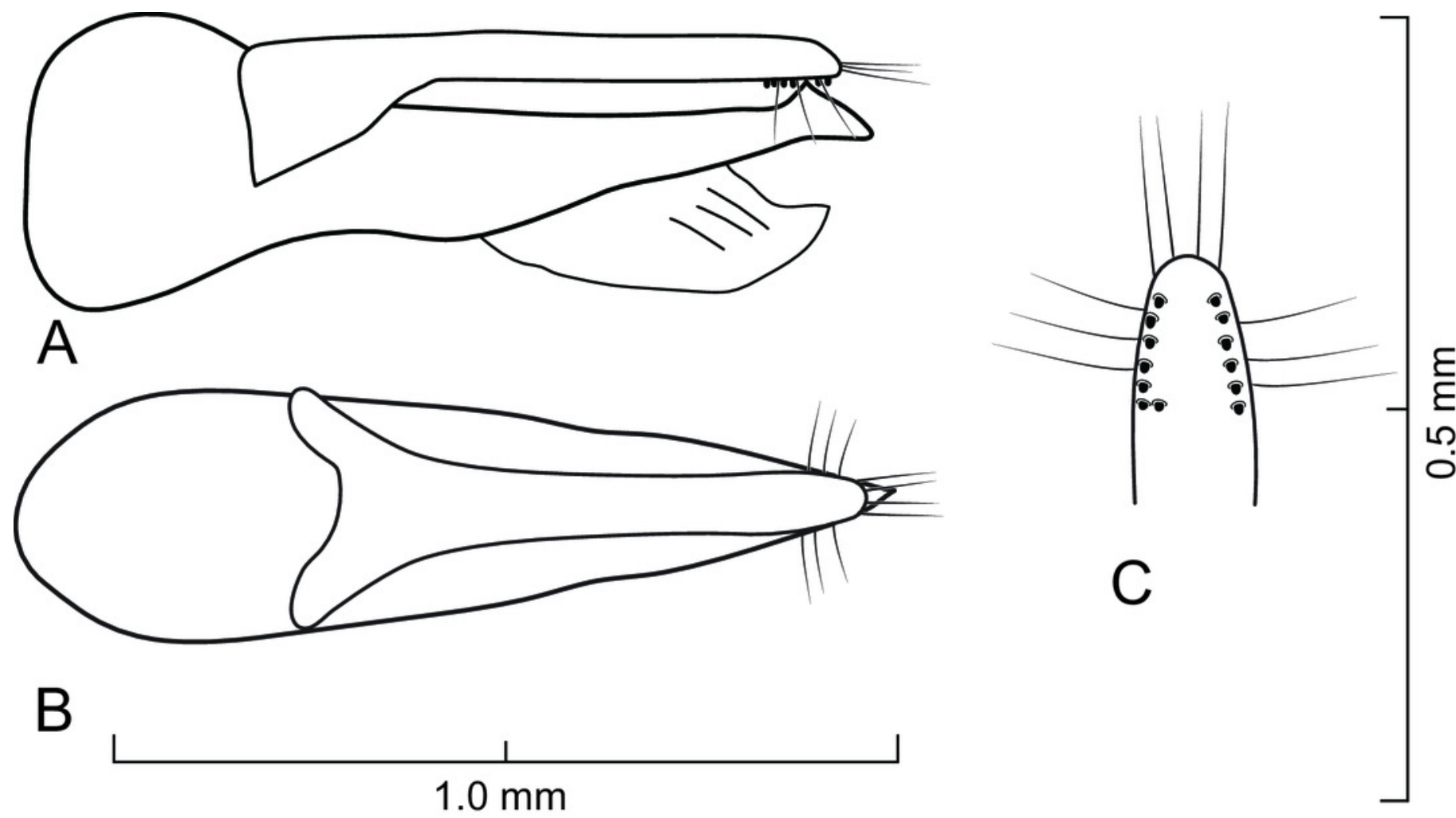
Figure 6

Aedeagus of Lendatus philothalpiformis Chatzimanolis.

(A) Lateral view. (B) Dorsal view. (C) Detail of paramere, ventral view.
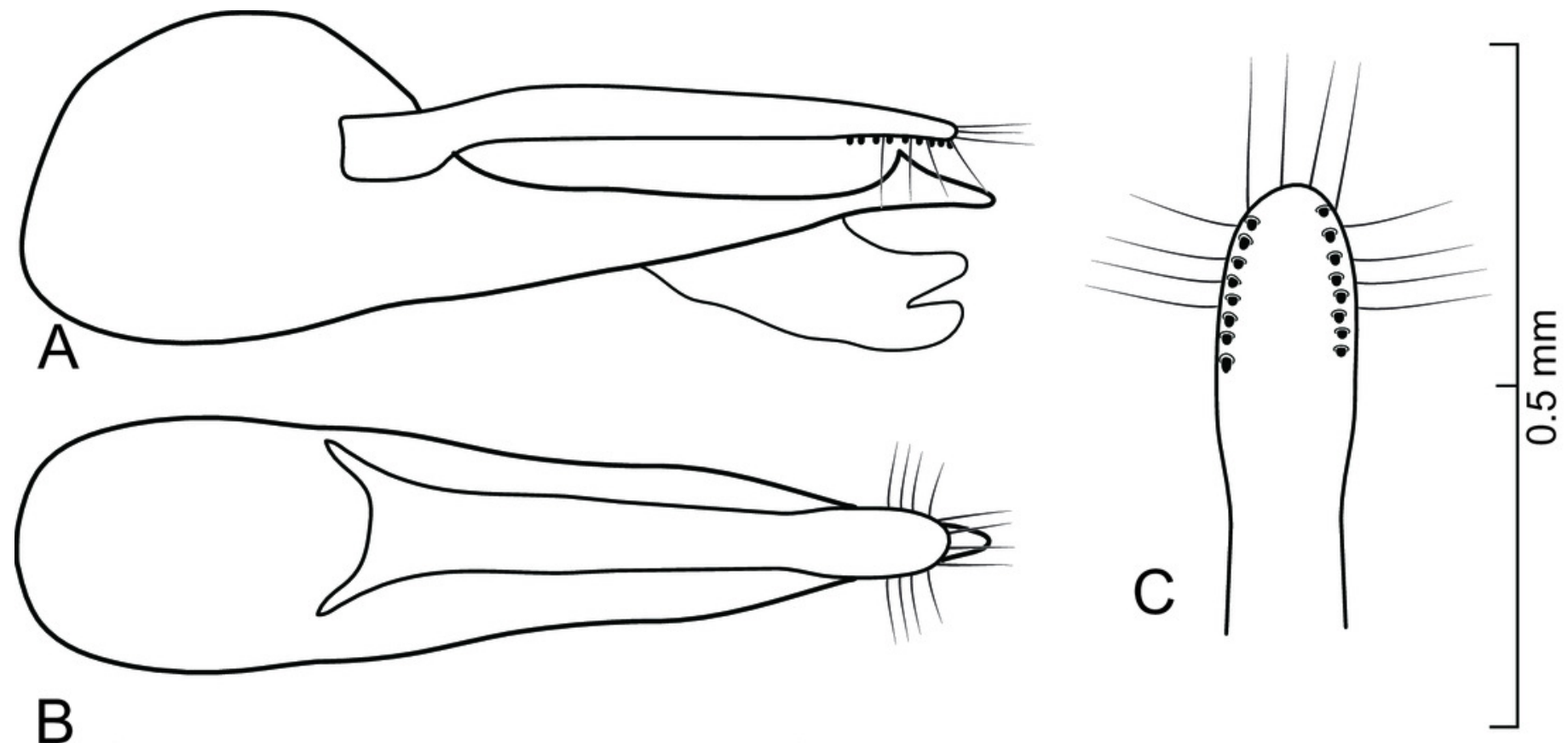

B

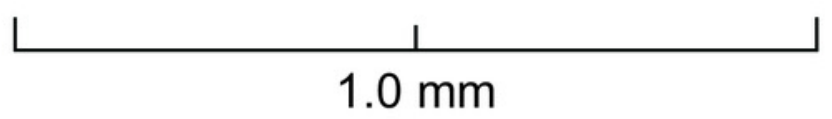


Figure 7

Aedeagus of Lendatus platys Chatzimanolis.

(A) Lateral view. (B) Dorsal view. (C) Detail of paramere, ventral view.

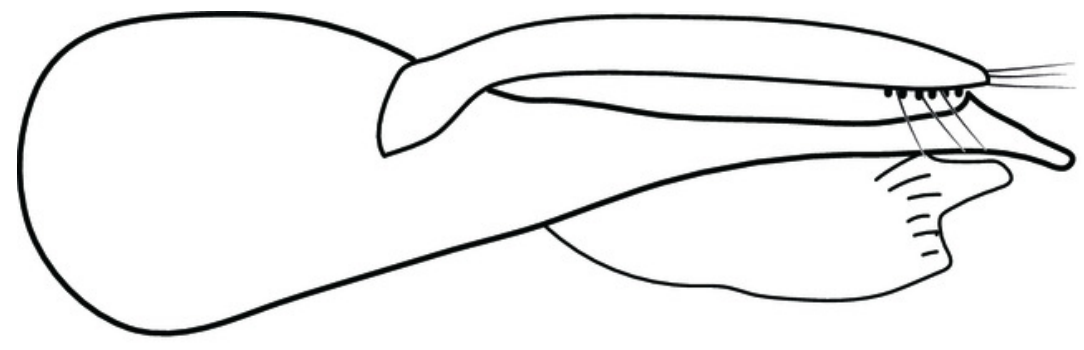

A
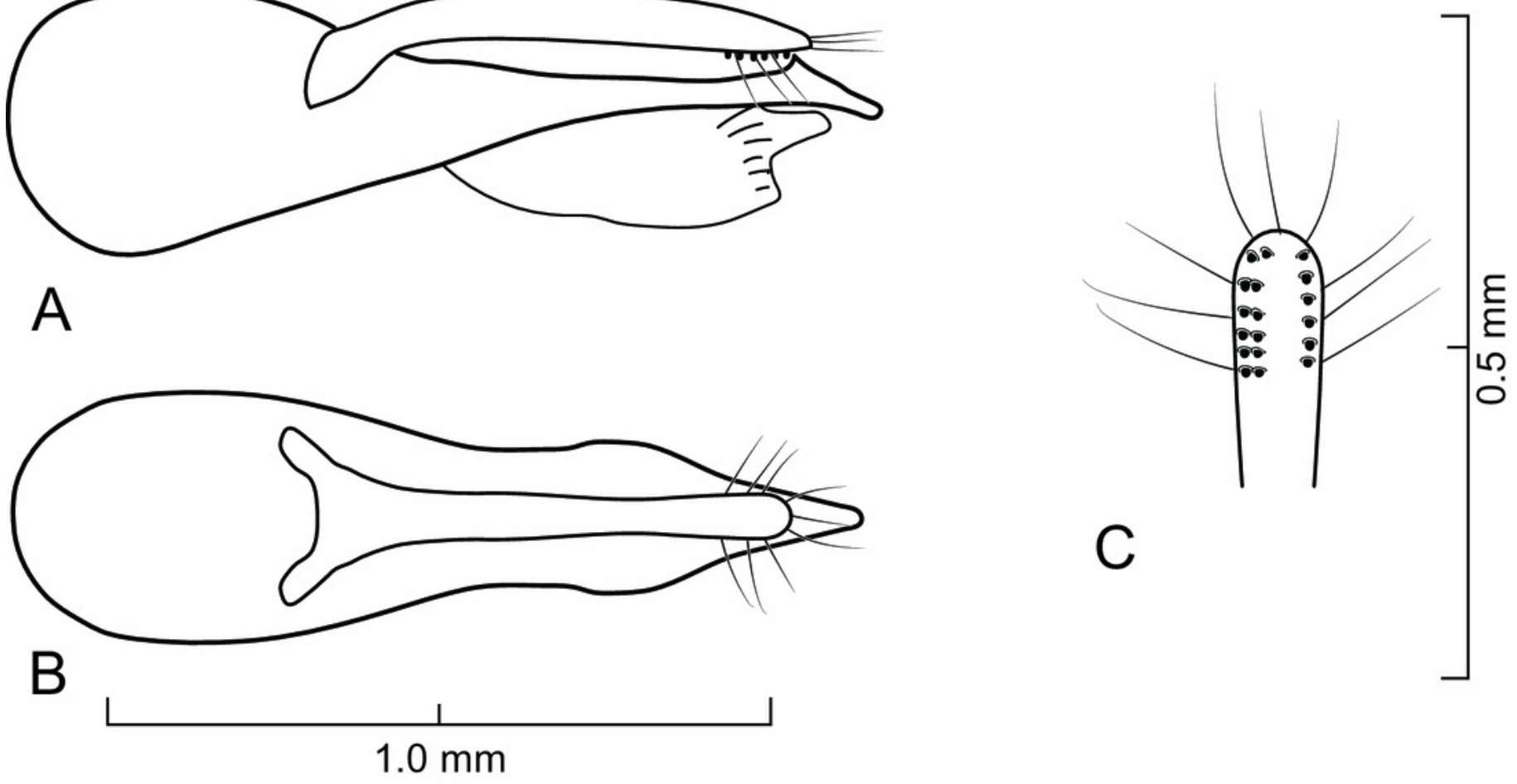
Figure 8

Distribution map of Lendatus bolivianus Chatzimanolis (square) and Lendatus platys Chatzimanolis (circles).

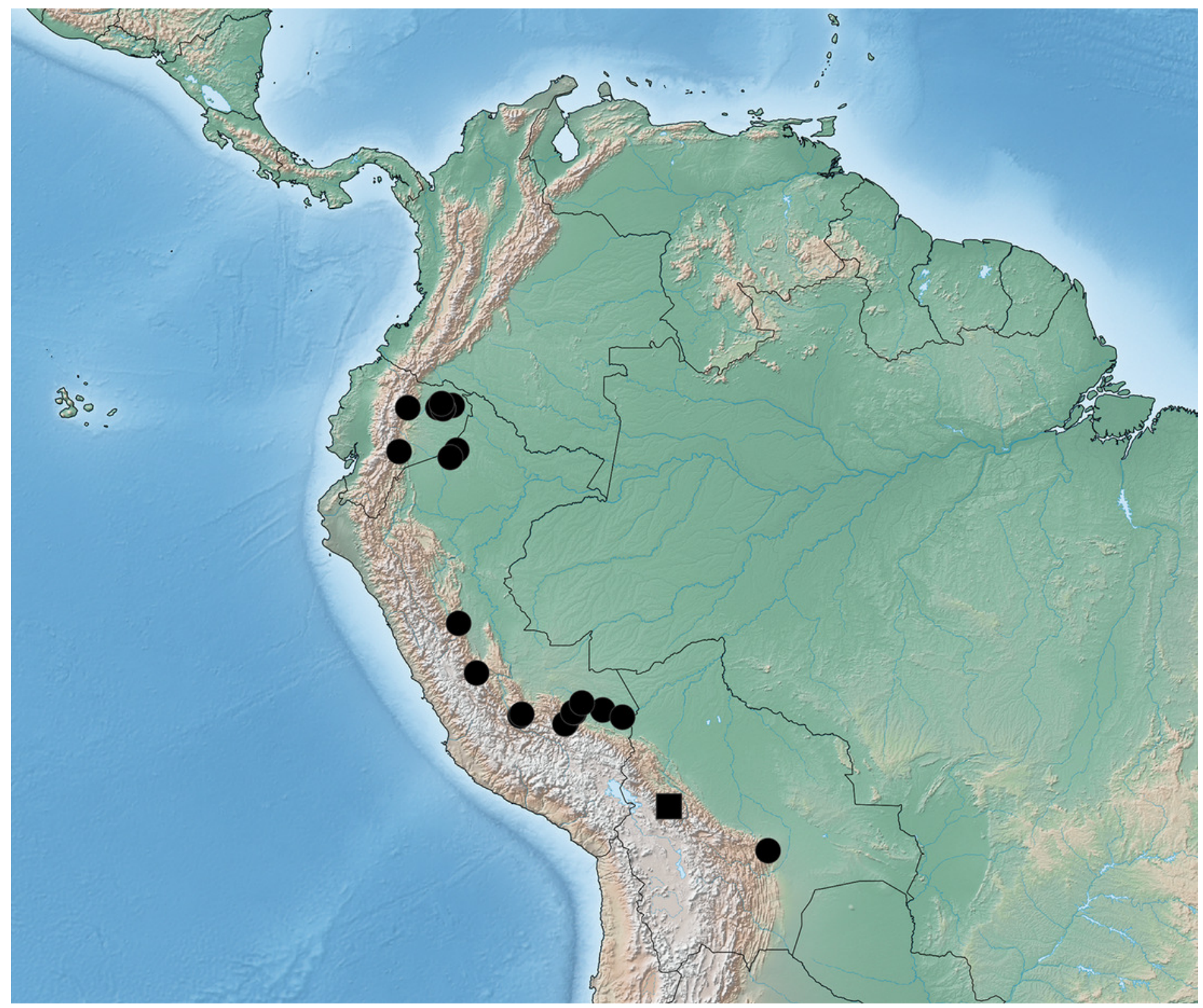


Figure 9

Distribution map of Lendatus philothalpiformis Chatzimanolis.

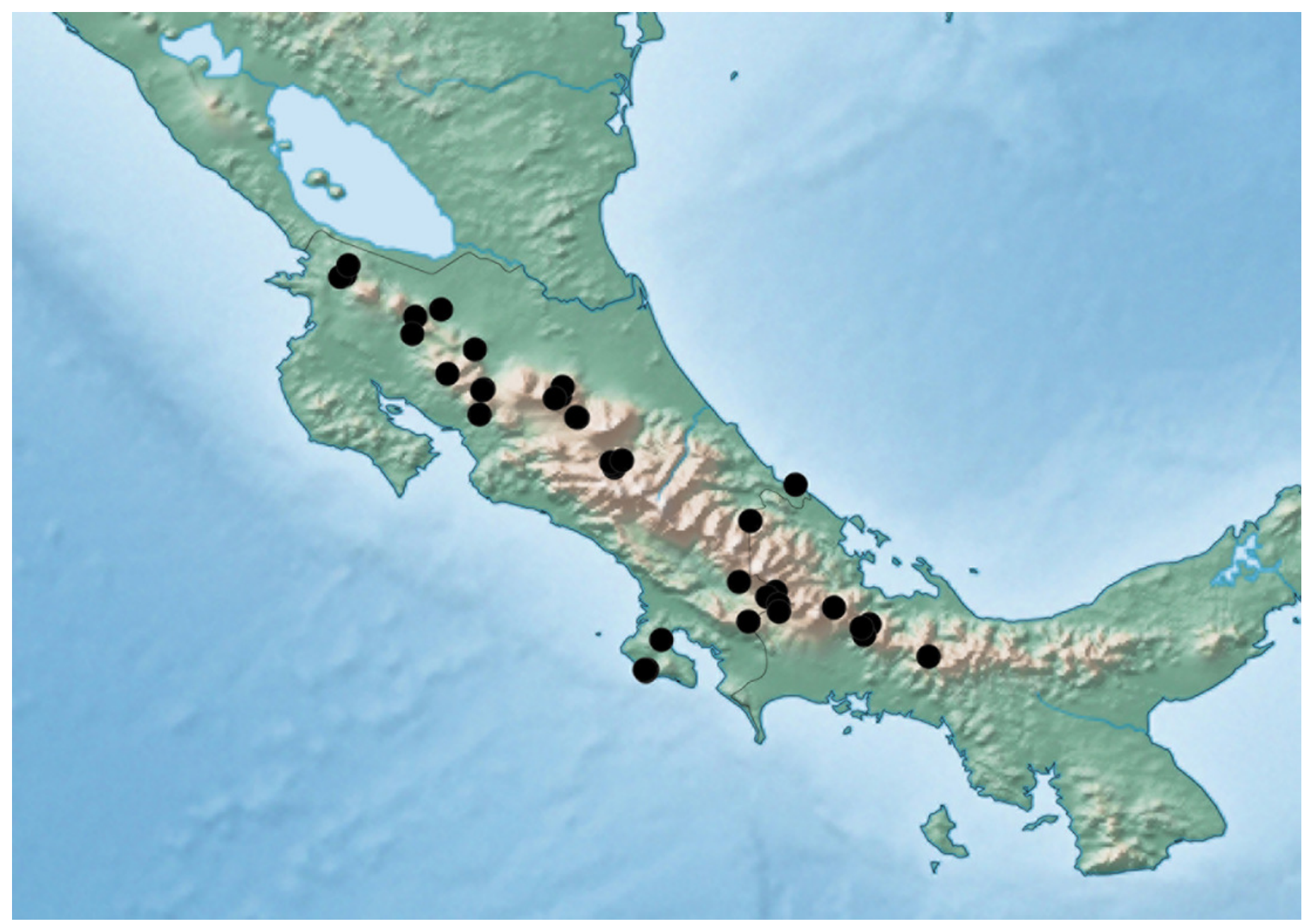

